\title{
Maconha: fator desencadeador de esquizofrenia?
}

\section{Cannabis: a triggering factor for schizophrenia?}

\author{
Vanessa Kelly de Oliveira ${ }^{1}$; Estefânia Gastaldello Moreira ${ }^{2}$
}

\section{Resumo}

A esquizofrenia acomete, aproximadamente, $1,1 \%$ da população. Atualmente, acredita-se que a esquizofrenia seja uma doença multifatorial, na qual deva existir uma predisposição genética, mas sejam necessários fatores ambientais desencadeadores para ela se manifestar. Entre os fatores ambientais sugeridos como desencadeadores, salienta-se o uso abusivo de algumas drogas psicotrópicas, incluindo a maconha. O objetivo deste trabalho foi fazer um levantamento bibliográfico dos trabalhos que estabelecem uma relação causal entre uso abusivo de maconha e manifestação de esquizofrenia. A análise da literatura mostrou que existem evidências crescentes, tanto epidemiológicas quanto biológicas, de que o uso de maconha pode desencadear o início da esquizofrenia, pelo menos em indivíduos predispostos.

Palavras-chave: Maconha. Abuso. Esquizofrenia. Doenças psiquiátricas.

\begin{abstract}
Schizophrenia has a prevalence of $1.1 \%$ in general population. It is believed to be a multifactorial disease in which a genetic predisposition may exist, but triggering environmental factors should also exist. Among the triggering environmental factors suggested, it is highlighted the use of some drugs, including cannabis (marijuana). The present report aimed to carry out a bibliographic review of the papers presenting a causal relationship between cannabis abuse and development of schizophrenia. The results showed that there is increasing evidence, both epidemiological and biological, that cannabis abuse may trigger schizophrenia, at least in predisposed individuals.

Key words: Cannabis. Marijuana. Excessive use. Schizophrenia. Mental Illness.
\end{abstract}

Enfermeira, concluinte do Curso de Especialização em Toxicologia Aplicada da UEL

2 Docente do Departamento de Ciências Fisiológicas, CCB, UEL. CEP 86051-980 - Londrina - PR - Fone: (43) 3371-4307.

E-mail: egmoreira@uel.br. 


\section{Introdução}

\section{Esquizofrenia}

A esquizofrenia é um distúrbio psiquiátrico relatado desde as civilizações mais remotas.

Estima-se que, aproximadamente, $1,1 \%$ da população seja acometido pela doença (REGIER et al., 1993), não havendo diferença na prevalência entre homens e mulheres (ROBINS ; REGIER, 1991). Entretanto, em homens, o início da doença geralmente ocorre no final da adolescência ou por volta dos 20 anos, enquanto em mulheres é mais comum o aparecimento ao redor dos 30 anos (ROBINS ; REGIER, 1991).

O diagnóstico baseia-se, exclusivamente, na história psiquiátrica e no exame do estado mental. Caracteriza-se por alterações do pensamento, da afetividade e do comportamento, trazendo, assim, conseqüências desastrosas aos portadores, bem como para as pessoas que convivem com os mesmos (BARON, 2001). Os sintomas tradicionais da esquizofrenia são classificados como positivos e negativos (STAHL, 2002). Os sintomas positivos incluem comportamentos bizarros, ilusões (geralmente paranóides), alucinações (principalmente auditivas, mas também visuais e tácteis) e as desordens do pensamento, que se caracterizam por distúrbios ideativos que podem levar a interpretações distorcidas da realidade. Os sintomas negativos incluem, principalmente, as alterações de afetividade, levando a perda de sentimentos e emoções. O paciente mostra ambivalência, reações emocionais inadequadas (i.e., impulsividade, agressividade, não reação a acontecimentos cotidianos), bem como desapego em relação às outras pessoas (embotamento afetivo). Assim, o comportamento torna-se abúlico (privado de vontade), sem iniciativa, notando-se uma enorme restrição de interesses e atividades. As relações sociais, logicamente, diminuem.

Atualmente, é amplamente aceito o envolvimento de alterações neurobiológicas na manifestação da esquizofrenia, havendo alterações morfológicas e funcionais no encéfalo do indivíduo (STAHL, 2002).
As alterações morfológicas foram as primeiras a serem observadas. Em 1899, Kraeplin, sugeriu que a esquizofrenia deveria ser uma "demência precoce", baseando-se na observação de distribuição neuronal anormal das camadas profundas do córtex dos pacientes. Mais recentemente, estudos têm mostrado outras alterações morfológicas em regiões corticais e subcorticais no encéfalo de esquizofrênicos, como a dilatação do sistema ventricular e falhas no desenvolvimento têmporo-límbico (BARON, 2001).

Funcionalmente, estudos sugerem que, na patogenia da esquizofrenia, há uma complexa alteração no funcionamento de muitas vias neurais e envolvimento de várias estruturas, como gânglios da base, córtex frontal, parietal e temporal (STAHL, 2002). Achados neuroquímicos sugerem que, no encéfalo de esquizofrênicos, há aumento da neurotransmissão dopaminérgica mesolímbica. Esta hipótese é fortalecida pelo fato de anfetaminas aumentarem a neurotransmissão dopaminérgica e poderem desencadear ou mimetizar o estado esquizofrênico e também pela ação das drogas antipsicóticas, que se baseia na diminuição da neurotransmissão dopaminérgica (STAHL, 2002). Entretanto, estudos mais recentes sugerem que a base neuroquímica seria, na realidade, um desequilíbrio entre as vias dopaminérgicas mesocortical e mesolímbica. Haveria hipofuncionamento da via mesocortical, responsável pelos sintomas negativos da esquizofrenia e hiperfuncionamento da via mesolímbica, responsável pelos sintomas positivos (KAHN ; DAVIS, 1995). Esta hipótese é reforçada pela observação clínica de que os antipsicóticos típicos, que diminuem a neurotransmissão dopaminérgica encefálica, controlam os sintomas positivos da esquizofrenia, mas exacerbam os negativos, promovendo a chamada síndrome deficitária induzida pelos antipsicóticos típicos (STAHL, 2002).

Alterações na neurotransmissão glutamatérgica também têm implicações na patogenia da esquizofrenia (OLNEY, 1988; PIETRASZEK, 2003). A hipótese glutamatérgica foi sugerida por Olney 
(1988) após a observação de relação positiva entre o bloqueio da função dos receptores glutamatérgicos NMDA pela droga fenciclidina (PCP) e o aparecimento de sintomas psicóticos. Ainda mais, há relatos de diminuição dos níveis de glutamato, com o conseqüente aumento na expressão dos seus receptores (up regulation) no córtex de pacientes esquizofrênicos (KOLB ; WHISHAW, 2003).

Embora o substrato neurobiológico da esquizofrenia tenha sido bastante estudado, a etiologia dessa doença ainda não é completamente compreendida. Acredita-se que existam fatores genéticos e/ou ambientais que desencadeiam as alterações morfológicas e funcionais no encéfalo do indivíduo. Atualmente, acredita-se que a esquizofrenia seja uma doença multifatorial, na qual deva existir uma predisposição genética, mas sejam necessários fatores ambientais desencadeadores para ela se manifestar (BROOME et al., 2005). O padrão de herança é complexo e acredita-se que existam múltiplos genes de susceptibilidade para a esquizofrenia, como aqueles envolvidos na expressão da neuroregulina, disbindina, catecol-Ometiltransferase e receptor serotonérgico $5-\mathrm{HT}_{2 \mathrm{~A}}$, todos importantes durante o neurodesenvolvimento (CORFAS; ROY; BUXBAUM, 2004; PIRJO et al., 2005). É interessante notar que várias anormalidades estruturais encefálicas foram descritas em adolescentes e adultos jovens com risco genético para esquizofrenia (KESHAVAN et al., 2002; LAWRIE et al., 1999, 2001).

Entre os fatores ambientais relacionados como desencadeadores da esquizofrenia, salientam-se tanto os intra-uterinos quanto os extra-uterinos. Entre os fatores intra-uterinos têm sido citadas infecções virais (influenza, rubéola, poliovírus, herpes) e bacterianas (BROWN et al., 2000; BUKA et al., 2001; SUVISAARI et al., 1999); desnutrição materna (SUSSER; LIN, 1992; SUSSER et al., 1996); complicações obstétricas como diabete gestacional, pré-eclâmpsia, incompatibilidade de $\mathrm{Rh}$, atonia uterina (CANNON; JONES; MURRAY, 2002); estresse emocional materno (KINNEY, 2001). Dentre os fatores extra-uterinos, temos o ambiente familiar nãofuncional (TIENARI et al., 1994); estresse emocional (KINNEY, 2001); uso de drogas psicotrópicas, como cocaína, anfetamina e maconha (EATON; MORTENSEN; FRYDENBERG, 2000).

\section{Maconha como Desencadeadora de Esquizofrenia}

A maconha é a droga ilícita mais consumida mundialmente. No Brasil, dados do Centro Brasileiro de Informações sobre Drogas Psicotrópicas (CEBRID) mostram que 6,9\% dos dependentes químicos usam maconha, ficando atrás somente do álcool e tabaco (CARLINI et al., 2002). Estima-se que $9 \%$ dos adolescentes já usaram maconha pelo menos uma vez na vida. Um dado que chama a atenção é que a idade do início do uso tem diminuído (CARLINI et al.; 2002).

A maconha é classificada como um perturbador do Sistema Nervoso Central, pois promove desorientação espacial e temporal e alterações do tato, visão e audição. Causa dependência e tolerância, mas tem baixa letalidade (SEIBEL; TOSCANO JUNIOR, 2004).

O primeiro relato de uso de maconha como possível desencadeador de esquizofrenia data de 1962 (ZAL'TSMAN; LENSKII, 1962). Entretanto, por anos a co-morbidade entre esquizofrenia e substâncias de abuso provocou controvérsias. Alguns autores acreditam em uma relação de causalidade, ou seja, o abuso de substâncias desencadearia esquizofrenia, pelo menos em indivíduos com predisposição (MUESER et al., 1990). Outros acreditam no modelo da auto-medicação, ou seja, pacientes esquizofrênicos usariam drogas de abuso para evitar o desconforto dos sintomas da doença ou do tratamento farmacológico (HAMBRECHT; HAFNER, 2000). E ainda há outros que acreditam que a co-morbidade poderia ser uma mera coincidência de duas desordens psiquiátricas (dependência e esquizofrenia), que têm picos de início coincidentes (adolescência e início da vida adulta para 
homens) e compartilham a mesma etiologia (fatores genéticos comuns, desregulação do sistema dopaminérgico de reforço e recompensa). Desse modo, não há nenhuma relação de causalidade entre dependência e esquizofrenia (CHAMBERS; KRYSTAL; SELF, 2001).

O primeiro estudo a investigar a hipótese do modelo causal foi um estudo epidemiológico sueco do tipo caso-controle, que acompanhou 50.000 jovens durante 15 anos (ANDREASSON et al., 1987). Este estudo sugeriu uma correlação positiva e dosedependente entre o início precoce de uso de maconha e o diagnóstico posterior de esquizofrenia em homens jovens. Em 2002, Zammit et al., revisaram a análise desta população ajustando-a para corrigir fatores de confusão como coeficiente de inteligência, fatores sócio-demográficos e uso de outras drogas. Eles encontraram que, para os indivíduos usuários somente de maconha, a relação dose-resposta permaneceu significativa com um risco aumentado de 1,5 vez. Para as pessoas que haviam usado maconha mais de 50 vezes, o risco subiu para 3,1 vezes.

Em 1993, um estudo sueco investigou 112 pacientes com diagnóstico de esquizofrenia e uso de maconha. Em 69\% dos pacientes, havia ocorrido abuso de maconha pelo menos um ano antes do início dos sintomas psicóticos. A doença precedeu o uso em apenas $11 \%$ dos casos (ALLEBECK; ADAMSSON; ENGSTROM, 1993).

Em 1994, um estudo holandês mostrou que em 23 dos 24 casos estudados o uso de maconha havia precedido o início dos primeiros sintomas psicóticos em pelo menos um ano (LINSZEN; DINGEMANS; LENIOR, 1994).

Arseneault et al. (2002) apresentaram dados de um estudo epidemiológico prospectivo que mostrava associação entre o uso de maconha entre adolescentes e a psicose em fases posteriores da vida. Os indivíduos que usavam maconha aos 15 anos de idade apresentaram maior incidência de sintomas da esquizofrenia, quando comparados com os indivíduos do grupo controle. Ainda mais, a incidência de diagnóstico para esquizofrenia foi 4 vezes maior neste grupo. Depois deste estudo, foram publicados três outros grandes estudos longitudinais em Israel, Nova Zelândia e Holanda, e eles evidenciavam a associação entre o uso de maconha e a manifestação posterior de psicose (FERGUSSON; HORWOOD; SWAIN-CAMPBELL, 2003; VAN OS et al., 2002; WEISER et al., 2003).

Após o ano de 2000, o modelo causal ganhou força (ARSENEAULT et al., 2004; SMIT; BOLIER; CUIJPERS, 2004), provavelmente pelos avanços ocorridos na neurobiologia do sistema endocanabinóide. A partir deste ano, revisões sistemáticas dos trabalhos epidemiológicos publicados até então passaram a ser feitas de modo a avaliar a força das evidências.

Arseneault et al. (2004) mostraram que o uso de maconha dobrava o risco para esquizofrenia e estimaram que a abolição do uso de maconha traria uma redução de, aproximadamente, $8 \%$ na incidência de esquizofrenia.

Henquet et al. (2005) reuniram sete estudos prospectivos publicados entre 2002 e 2005 que examinavam a associação entre uso de maconha e desenvolvimento de psicose e os submeteram a um estudo de meta-análise. $\mathrm{O}$ resultado revelou que havia uma associação positiva entre os dois fatores com um risco relativo ao redor de 2. Embora os autores tenham reconhecido que este valor não era tão alto, eles argumentam que, como o uso de maconha é bastante prevalente na população jovem, que é o grupo de maior risco para desenvolvimento de psicose, o dado tornava-se bastante relevante.

Semple, Mcintosh e Lawrie (2005) fizeram uma revisão sistemática da literatura e incluíram na metaanálise somente os estudos que examinaram claramente a associação entre uso de maconha e esquizofrenia ou psicose esquizofrênica, mas não com sintomas psicóticos. Estes autores encontraram que o uso de maconha era um fator de risco e triplicava a chance de aparecimento de esquizofrenia. 
Além das evidências epidemiológicas de que o uso de maconha possa de fato atuar como desencadeador da esquizofrenia, algumas evidências biológicas também existem.

O principal canabinóide responsável pelos efeitos psicoativos da maconha é o delta-9tetrahidrocanabinol (THC). Atualmente, sabe-se que ele atua como agonista de receptores canabinóides (CB), presentes tanto no encéfalo (CB1) quanto em estruturas do sistema imune (CB2). Estes receptores pertencem à família de receptores acoplados à proteína $\mathrm{G}$ inibitória. Quando ativados, estes receptores levam a diminuição de AMP cíclico intracelular resultando em fechamento de canais de cálcio e abertura de canais de potássio e, portanto, inibição neuronal (BREIVOGEL; CHILDERS, 1998). O THC mimetiza a ação de substâncias endógenas chamadas de endocanabinóides, isoladas na década de 1990. Dois endocanabinóides foram isolados: a anandamida (DEVANE et al., 1992) e o 2-aracdonil-glicerol (MECHOULAM; HANUS; MARTIN, 1994). Acredita-se que os endocanabinóides sejam sintetizados por células póssinápticas e ajam como mensageiros retrógrados para modular a liberação de outros neurotransmissores (KREITZER; REGEHR, 2001).

Os receptores CB1 estão amplamente distribuídos no cerebelo, hipocampo, amígdala, gânglios da base e córtex (GLASS; FAULL; DRAGUNOW, 1997b). Esta distribuição regional está de acordo com os efeitos centrais conhecidos dos canabinóides em alterar o humor e o estado emocional, interferir na memória de curtoprazo, promover desorientação espacial e temporal (BREIVOGEL; CHILDERS, 1998).

As principais evidências biológicas de que o sistema canabinóide esteja envolvido na patogenia da esquizofrenia são:

a) Anatomicamente, os receptores canabinóides estão distribuídos em regiões que têm sido implicadas na esquizofrenia, como córtex pré-frontal, gânglios da base, hipocampo e córtex cingulado anterior (GLASS; FAULL; DRAGUNOW, 1997b). b) Níveis elevados de endocanabinóides (anandamida e palmitiletanolamida) foram encontrados no líquido céfalo-raquidiano de 10 pacientes diagnosticados com esquizofrenia (LEWEKE et al., 1999a).

c) Níveis aumentados de receptores CB1 no Sistema Nervoso Central, incluindo o córtex cingulado anterior têm sido descritos em esquizofrênicos (DEAN et al., 2001; ZAVITSANOU; GARRICK; HUANG, 2004). $O$ córtex cingulado anterior é importante para a cognição, principalmente aquela relacionada à motivação e atenção (BUSH; LUU; POSNER, 2000). Portanto, as alterações no sistema endocanabinóide desta região podem estar relacionadas com os sintomas negativos da esquizofrenia.

d) Abuso de maconha piora os sintomas psicóticos em pacientes esquizofrênicos (ANDREASSON et al., 1987; LINSZEN; DINGEMANS; LENIOR, 1994).

e) Similaridades entre prejuízos cognitivos que ocorrem nas psicoses e os efeitos farmacológicos da maconha têm sido identificados (EMRICH; LEWEKE; SCHNEIDER, 1997; LEWEKE et al., 1999b).

f) Existem interações funcionais entre dopamina e canabinóides. As drogas que bloqueiam receptor dopaminérgico $\mathrm{D}_{2}$ diminuem os sintomas positivos da esquizofrenia e estudos de microdilálise mostraram que estímulo de $\mathrm{D}_{2}$ no estriado aumenta a liberação de anandamida (GIUFFRIDA et al., 1999). O significado fisiológico das interações dopamina-anandamida não é bem compreendido, mas estudos comportamentais mostram que in vivo ocorrem interações (GLASS; BROTCHIE; MANEUF, 1997a).

Pelo que se expôs, fica claro que o sistema canabinóide de fato está implicado na patogenia da esquizofrenia e a ativação deste sistema pelo uso de maconha poderia ser a base neurobiológica que explicaria o desencadeamento de esquizofrenia pelo uso de maconha. Estudos experimentais mostraram que o tratamento com THC por duas semanas reduziu a neurotransmissão dopaminérgica no córtex frontal 
(JENTSCH et al., 1998) e aumentou a neurotransmissão dopaminérgica mesolímbica (TANDA; PONTIERI; DI CHIARA, 1997). Como foi anteriormente comentado, este desbalanço também está presente no encéfalo do paciente esquizofrênico (STAHL, 2002).

Um outro possível mecanismo biológico que poderia explicar a associação entre uso de maconha e psicose é a sensibilização à dopamina, observada após uso regular de algumas drogas de abuso, incluindo a maconha. Sabe-se que os indivíduos que usam regularmente maconha, tornam-se progressivamente mais vulneráveis às aberrações cognitivas e sensoriais induzidas pela dopamina e passíveis de progredir para sintomas psicóticos (HOWES et al., 2004).

É interessante notar que alguns estudos mostraram que, quanto mais cedo se dá o início do uso de maconha, maior é o risco de desenvolvimento de psicose (ARSENEAULT et al., 2002; FERGUSSON; HORWOOD; SWAINCAMPBELL, 2003; STEFANIS et al., 2004). Esta observação clínica é corroborada por estudos experimentais. Sabe-se que o sistema endocanabinóide está envolvido no neurodesenvolvimento, e estudos experimentais mostraram que a administração prolongada de THC a ratos durante a puberdade induziu mudanças comportamentais e cognitivas que não foram observadas em animais expostos durante a fase adulta (SCHNEIDER; KOCK, 2003). Ainda mais, um estudo de neuroimagem mostrou que tanto homens quanto mulheres que iniciaram o uso de maconha antes de 17 anos apresentaram uma menor porcentagem de matéria cinzenta cortical do que aqueles que iniciaram posteriormente (WILSON et al., 2000). Em conjunto, os dados sugerem que a adolescência pode ser um período de vulnerabilidade do encéfalo para os efeitos adversos da maconha.

\section{Conclusão}

Existem evidências crescentes, tanto epidemiológicas quanto biológicas, de que o uso de maconha pode desencadear o início da esquizofrenia, pelo menos em indivíduos predispostos. Se o uso inadequado pode desencadear a doença em indivíduos sem predisposição ainda é uma dúvida.

\section{Referências}

ALLEBECK, P.; ADAMSSON, C.; ENGSTROM, A. Cannabis and schizophrenia: a longitudinal study of cases treated in Stockholm County. Acta Psychiatrica Scandinavica, Copenhagen, v.88, n.1, p.21-24, 1993.

ANDREASSON, S.; ENGSTROM, A.; ALLEBECK, P.; RYDBERG, U. Cannabis and schizophrenia - a longitudinalstudy of Swedish conscripts. Lancet, London, v.2, n.8574, p.1483-1486, 1987.

ARSENEAULT, L.; CANNON, M.; POULTON, R.; MURRAY, R.; CASPI, A.; MOFFITT, T. E. Cannabis use in adolescence and risk for adult psychosis: longitudinal prospective study. British Medical Journal, London, v.325, n.7374, p.1212-1213, 2002.

ARSENEAULT, L.; CANNON, M.; WITTON, J.; MURRAY, R. M. Causal association between cannabis use and psychosis: examination of the evidence. British Journal of Psychiatry, London, v.184, p.110-117, feb.2004.

BARON, A. L. D. Diferenças de efeitos agudos sobre a perfusão regional cerebral entre neurolépticos convencionais (haloperidol) e de nova geração (olanzapina) em portadores de esquizofrenia. Rio Grande do Sul: UFRGS, 2001.

BROOME, M. R.; WOOLEY, J. B.; TABRAHAM, P.; JOHNS, L. C.; BRAMON, E.; MURRAY, G. K.; PARIANTE, C.; McGUIRE, P. K.; MURRAY, R. M. What causes the onset of psychosis? Schizophrenia Research, Amsterdam, v.79, n.1, p.23-34, 2005.

BROWN, A. S.; COHEN, P.; GREENWALD, S.; SUSSER, E. Nonaffective psychosis after prenatal exposure to rubeola. American Journal of Psychiatry, Arlington, v.157, n.3, p.438-443, 2000.

BREIVOGEL, C. S.; CHILDERS, S. R. The functional neuroanatomy of brain cannabinoid receptors. Neurobiology of Disease, Orlando, v.5, n.6, p.417-431, 1998. 
BUKA, S. L.; TSUANG, M. T.; TORREY, E. F.; KLEBANOFF, M. A.; BERNESTEIN, D.; YOLKEN, R. H. Maternal infections and subsequent psychosis among offspring. Archives of General Psychiatry, Chicago, v.58, n.11, p.1032-1037, nov.2001.

BUSH, G.; LUU, P.; POSNER, M. I. Cognitive and emotional influences in anterior cingulated cortex. Trends in Cognitive Sciences, Kidlington, v.4, n.6, p.215-222, 2000.

CANNON, M.; JONES, P. B.; MURRAY, R. M. Obstetric complications and schizophrenia: historical and metaanalytic review. American Journal of Psychiatry, Arlington, v.159, n.7, p.1080-1092, 2002.

CARLINI, E. A.; GALDUROZ, J. C. F.; NOTO, A. R.; NAPPO, S. A. Levantamento domiciliar sobre o uso de drogas psicotrópicas no Brasil: estudo envolvendo as 107 maiores cidades do país, 2001. São Paulo: Secretaria Nacional Antidrogas; 2002.

CHAMBERS, R. A.; KRYSTAL, J. H.; SELF, D. W. A neurobiological basis for substance abuse comorbidity in schizophrenia. Biological Psychiatry, New York, v.50, n.2, p.71-83, 2001.

CORFAS, G.; ROY, K.; BUXBAUM, J.D. Neuregulin 1-erbB signaling and the molecular/cellular basis of schizophrenia. Nature Neuroscience, New York, v.7, n.6, p.575-580, 2004.

DEAN, B.; SUNDRAM, S.; BRADBURY, R.; SCARR, E.; COPOLOV, D. Studies on $\left[{ }^{3} \mathrm{H}\right] \mathrm{CP}-55940$ binding in the human central nervous system: regional specific changes in density of CB-1 receptors associated with schizophrenia and cannabis use. Neuroscience, Oxford, v.103, n.1, p.9$15,2001$.

DEVANE, W. A.; HANUS, L.; BREUER, A.; PERTWEE, R. G.; STEVENSON, L. A.; GRIFFIN, G.; GIBSON, D.; MANDELBAUM, A.; ETINGER, A.; MECHOULAM, R. Isolation and structure of a brain constituent that binds to the CB receptor. Science, Washington, v.258, n.5090, p.1946-1949, 1992.

EATON, W. W.; MORTENSEN, P. B.; FRYDENBERG, M. Obstetric factors, urbanisation and psychosis. Schizophrenia Research, Amsterdam, v.43, n.2-3, p.117123, 2000.

EMRICH,H. M.; LEWEKE, F. M.; SCHNEIDER, U. Towards a cannabinoid hypothesis of schizophrenia: cognitive impairments due to dysregulation of the endogenous cannabinoid system. Pharmacology, Biochemistry and Behavior, Phoenix, v.56, n.4, p.803-807, 1997.

FERGUSSON, D. M.; HORWOOD, L. J.; SWAINCAMPBELL, N. R. Cannabis dependence and psychotic symptons in young people. Psychological Medicine, London, v.33, n.1, p.15-21, 2003.
GIUFFRIDA, A.; PARSONS, L.H.;KERR, T. M.; RODRIGUEZ DE FONSECA, F.; NAVARRO, M.; PIOMELLI, D. Dopamine activation of endogenous cannabinoid signaling in dorsal striatum. Nature Neursocience, New York, v.2, n.4, p.358-363, 1999.

GLASS, M.; BROTCHIE, J. M.; MANEUF, Y. P. Modulation of neurotransmission by cannabinoids in the basal ganglia. European Journal of Neuroscience, Oxford, v.9, n.2, p.199-203, 1997a.

GLASS, M.; FAULL, R. L. M.; DRAGUNOW, M. Cannabinoid receptors in the human brain: a detailed anatomical and quantitative autoradiographic study in the fetal, neonatal and adult human brain. Neuroscience, Oxford, v.77, n.2, p.299-318, 1997 b.

HAMBRECHT, M.; HAFNER, H. Cannabis, vulnerability and the onset of schizophrenia: an epidemiological perspective. Australian and New Zealand Journal of Psychiatry, Melbourne, v. 34, n.3, p.468-475, 2000.

HENQUET, C.; MURRAY, R.; LINSZEN, D.; VAN OS, J. The environment and schizophrenia: the role of cannabis use. Schizophrenia Bulletin, Rockville, v.31, n.3, p.608612, 2005.

HOWES, O. D.; McDONALD, C.; CANNON, M.; ARSENEAULT, L.; BOYDELL, J.; MURRAY, R. M. Pathways to schizophrenia: the impact of environmental factors. International Journal of Neuropsychopharmacology, Cambridge, v.7, Supl.1, p.S7S13, 2004.

JENTSCH, J. D.; VERRICO, C. D.; LE, D.; ROTH, R. H. Repeated exposure to delta 9-tetrahydrocannabinol reduces prefrontal cortical dopamine metabolism in the rat. Neuroscience Letters, Amsterdam, v.246, n.3, p.169172, 1998.

KAHN, R. S.; DAVIS, K. L. New developments in dopamine and schizophrenia. In: BLOOM, F. E.; KUPFER, D. J. Psychopharmacology: The Fourth Generation of Progress. New York: Raven Press, 1995. p.1193-1201.

KESHAVAN, M. S.; DICK, E.; MANKOWSKI, I.; HARENSKI, K.; MONTROSE, D. M.; DIWADKAR, V.; DEBELLIS, M. Decreased left amygdala and hippocampal volumes in young offspring at risk for schizophrenia. Schizophrenia Research, Amsterdam, v.58, n.2-3, p.173183, 2002.

KINNEY, D. K. Prenatal stress and risk for schizophrenia. International Journal of Mental Health, Carlton, v.29, n.4, p.62-72, 2001.

KOLB, B.; WHISHAW, I. Q. Fundamentals of Human Neuropsychology. New York: Worth Publishers, 2003. 
KRAEPLIN, E. Psychiatry: a textbook for students and physicians. [Canton: Science History Publication], 1899.

KREITZER, A. C.; REGEHR, W. G. Retrograde inhibition of presynaptic calcium influx by endogenous cannabinoids at excitatory synapses onto Purkinje cells. Neuron, Cambridge, v.29, n.3, p.717-727, 2001.

LAWRIE, S. M.; WHALLEY, H. C.; KESTELMAN, J. N.; ABUKMEIL, S. S.; BYRNE, M.; HODGES, A., RIMMINGTON, J. E.; BEST, J. J.; OWENS, D. G.; JOHNSTONE, E. C. Magnetic resonance imaging of brain people at high risk of developing schizophrenia. Lancet, London, v.353, n.9146, p.30-33, 1999.

LAWRIE, S. M.; WHALLEY, H. C; ABUKMEIL, S. S.; KESTELMAN, J. N.; DONNELLY, L.; MILLER, P.; BEST, J. J.; OWENS, D. G.; JOHNSTONE, E. C. Brain structure, genetic liability, and psychotic symptoms in subjects at high risk of developing schizophrenia. Biological Psychiatry, New York, v.49, n.10, p.811-823, 2001.

LEWEKE, F. M.; GIUFFRIDA, A.; WURSTER, U.; EMRICH, H. M.; PIOMELLI, D. Elevated endogenous cannabinoids in schizophrenia. NeuroReport, Oxford, v.10, n.8, p.1665-1669, 1999a.

LEWEKE, F. M.; SCHNEIDER, U.; THIES, M.; MUNTE, T. F.; EMRICH, H. M. Effects of synthetic delta(9)tetrahydrocannabinol on binocular depth inversion of natural and artificial objects in man. Psychopharmacology, Berlin, v.142, n.3, p.230-236, 1999b.

LINSZEN, D. H.; DINGEMANS, P. M; LENIOR, M. E. Cannabis abuse and the course of recent-onset schizophrenic disorders. Archives of General Psychiatry, Chicago, v.51, n.4, p.273-279, 1994.

MECHOULAM, R.; HANUS, L.; MARTIN, B. R. Search for endogenous ligands of the CB receptor. Biochemical Pharmacology, Oxford, v.48, n.8, p.1537-1544, 1994.

MUESER, K. T.; YARNOLD, P. R.; LEVINSON, D.F.; SINGH, H.; BELLACK, A. S.; KEE, K.; MORRISON, R. L.; YADALAM, K. G. Prevalence of substance abuse in schizophrenia: demographic and clinical correlates. Schizophrenia Bulletin, Rockville, v.16, n.1, p.31-56, 1990.

OLNEY, J. W. Endogenous excitotoxins and neuropathological disorders. In: LODGE D (Ed.). Excitatory Amino acids in health and disease. England: John Wiley \& Sons, 1988. p.337-351.

PIETRASZEK, M. Significance of dysfunctional glutamatergic transmission for the development of psychotic symptoms. Polish Journal of Pharmacology, Kraków, v.55, n.3, p.133-154, 2003.

PIRJO, M.; JUHA, V.; JONES, P. B.; MURRAY, G. K.; KOPONEN, H.; TIENARI, P.; MIETTUNEN, J.;
TANSKANEN, P.; WAHLBERG, K. E.; KOSKINEN, J.; LAURONEN, E.; ISOHANNI, M. Predictors of schizophrenia - a review. British Medical Bulletin, London, v.73-74, n.1, p.1-15, 2005.

REGIER, D. A.; NARROW, W. E.; RAE, D. S.; MANDERSCHEID, R. W.; LOCKE, B. Z.; GOODWIN, F. $K$. The de facto US mental and addictive disorders service system. Epidemiologic Catchment Area prospective 1-year prevalence rates of disorders and services. Archives of General Psychiatry, Chicago, v.50, n.2, p.85-94, 1993.

ROBINS, L. N.; REGIER, D. A. Psychiatric disorders in America: the epidemiologic catchment area study. New York: The Free Press, 1991.

SCHNEIDER, M.; KOCH, M. Chronic pubertal, but not adult chronic cannabinoid treatment impairs sensorimotor gating, recognition memory, and the performance in a progressive ratio task in adult rats. Neuropsychopharmacology, New York, v. 28, n.10, p.17601769, 2003.

SEIBEL, S. D.; TOSCANO Jr., A. Dependência de Drogas. São Paulo: Atheneu, 2004.

SEMPLE, D. M.; McINTOSH, A. M.; LAWRIE, S. M. Cannabis as a risk factor for psychosis: systematic review. Journal of Psychopharmacology, Thousand Oaks CA, v.19, n.2, p.187-194, 2005.

SMIT, F.; BOLIER, L.; CUIJPERS, P. Cannabis use and the risk of later schizophrenia: a review. Addiction, London, v.99, n.4, p.425-430, 2004.

STAHL, S. M. Psicofarmacologia: base neurocientífica e aplicações práticas. Rio de Janeiro: Medsi, 2002.

STEFANIS, N. C.; DELESPAUL, P.; HENQUET, C.; BAKOULA, C.; STEFANIS, C. N.; VAN OS, J. Early adolescent cannabis exposure and positive and negative dimensions of psychosis. Addiction, London, v.99, n.10, p.1333-1341, 2004.

SUSSER, E.; LIN, S. P. Schizophrenia after prenatal exposure to the Dutch Hunger Winter of 1944-1945. Archives of General Psychiatry, Chicago, v.49, n.2, p.983988, 1992.

SUSSER, E.; NEUGEBAUER, R.; HOEK, H. W.; BROWN, A. S.; LIN, S.; LABOVITZ, D.; GORMAN, J. M. Schizophrenia after prenatal famine. Archives of General Psychiatry, Chicago, v.53, n.1, p.25-31, 1996.

SUVISAARI, J.; HAUKKA, J.; TANSKANEN, A.; HOVI, T.; LONNQVIST, J. Association between prenatal exposure to poliovirus infection and adult schizophrenia. American Journal of Psychiatry, Arlington, v.156, n.7, p.1100-1102, 1999. 
TANDA, G.; PONTIERI, F.E.; DI CHIARA, G. Cannabinoid and heroin activation of mesolimbic dopamine transmission by a common mul opioid receptor mechanism. Science, Washington, v.276, 2084-2050, 1997.

TIENARI, P.; WYNNE, L. C.; MORING, J.; LAHTI, I.; NAARALA, M.; SORRI, A.; WAHLBERG, K. E.; SAARENTO, O.; SEITAMAA, M.; KALEVA, M. The Finnish adoptive family study of schizophrenia. Implications for family research. British Journal of Psychiatry, London, Suppl., n.23, p.20-26, 1994.

VAN OS, J.; BAK, M.; HANSSEN, M.; BIJL, R. V.; DE GRAAF, R. VERDOUX, H. Cannabis use and psychosis: a longitudinal population-based study. American Journal of Epidemiology, Tokyo, v.156, n.4, p.319-327, 2002.

WEISER, M.; REICHENBERG, A.; RABINOWITZ, J.; KAPLAN, Z.; CASPI, A.; YASVIZKY, R.; MARK, M; KNOBLER, H. Y.; NAHON, D.; DAVIDSON, M. Selfreported drug abuse in male adolescents with behavioral disturbances, and follow-up for future schizophrenia. Biological Psychiatry, New York, v.54, n.6, p.655-660, 2003.
WILSON, W.; MATHEW, R.; TURKINGTON, T.; HAWK, T.; COLEMAN, R. E.; PROVENZALE, J. Brain morphological changes and early marijuana use: a magnetic resonance and positron emission tomography study. Journal of Addiction Disease, v.19, n.1, p.1-22, 2000.

ZAL'TSMAN, G. I; LENSKII, G. P. On the effect of hashish on the psyche. Zdravookhranenie Kazakhstana, Almaty, v.22, n.9, p.30-35, 1962.

ZAMMIT, S.; ALLEBECK, P.; ANDREASSON, S.; LUNDBERG, I.; LEWIS, G. Self reported cannabis use as a risk factor for schizophrenia in Swedish conscripts of 1969: historical cohort study. British Medical Journal, London, v.325, n.7374, p.1199, 2002.

ZAVITSANOU, K.; GARRICK, T.; HUANG, X. F. Selective antagonist binding to $\mathrm{CB} 1$ receptors is increased in the anterior cingulate córtex in schizophrenia. Progress in Neuro-Psychopharmacology and Biological Psychiatry, Oxford, v.28, n.2, p.355-360, 2004. 
